\title{
NOTE ON THE $n$-DIMENSIONAL TEMPERED ULTRA-DISTRIBUTIONS
}

\author{
MORISUKE HASUMI
}

(Received July 1,1960)

In this note, we shall describe explicitly the duality in the space of tempered ultra-distributions of J. Sebastião e Silva in the Euclidean $n$-space. And, as an application, we shall prove a theorem on the multiplication of tempered ultradistributions.

I wish to express my hearty thanks to Prof. K. Yosida and Prof. J. Sebastião e Silva for reading the manuscript and giving me valuable remarks.

Notations : Let $R^{n}$ (resp. $C^{n}$ ) be the real (resp. complex) $n$-space whose generic points are denoted by $x=\left(x_{1}, \ldots ., x_{n}\right)$ (resp. $z=\left(z_{1}, \ldots, \ldots, z_{n}\right)$ ). We shall use the notations: (i) $x+y=\left(x_{1}+y_{1}, \ldots \ldots, x_{n}+y_{n}\right), \alpha x=\left(\alpha x_{1}, \ldots \ldots, \alpha x_{n}\right)$; (ii) $x \geqq 0$ means $x_{1} \geqq 0, \ldots \ldots, x_{n} \geqq 0$; (iii) $x \cdot y=\sum_{j=1}^{n} x_{j} y_{j}$ and (iv) $|x|$ $=\sum_{j=1}^{n}\left|x_{j}\right|$.

Let $p$ be a system of integers $\geqq 0,\left(p_{1}, \ldots, \ldots, p_{n}\right)$. We shall denote by $|p|$ the sum $\sum_{j=1}^{n} p_{j}$ and by $D^{p}$ the partial differential operator $\partial^{p_{1}+\cdots+p_{n}} / \partial x_{1}{ }^{p_{1}} \ldots$ $\ldots \partial x_{n}{ }^{p_{n}}$. We put, for any integer $k \geqq 0, \partial^{k} / \partial x^{k}=\partial^{n k} / \partial x_{1}{ }^{k} \ldots \ldots \partial x_{n}{ }^{k} . \quad p+q$ is the system of integers $\left(p_{1}+q_{1}, \ldots \ldots, p_{n}+q_{n}\right) . p \geqq q$ means $p_{1} \geqq q_{1}, \ldots \ldots, p_{n} \geqq q_{n}$. Moreover, $x^{p}=x_{1}{ }^{p_{1}} \ldots \ldots x_{n}{ }^{p_{n}}$ and $x^{k}=x_{1}{ }^{k} \ldots \ldots x_{n}{ }^{k}$ ( $k$ an integer). For $p \geqq q$, put $\left(\begin{array}{l}p \\ q\end{array}\right)=\left(\begin{array}{l}p_{1} \\ q_{1}\end{array}\right) \ldots \ldots\left(\begin{array}{l}p_{n} \\ q_{n}\end{array}\right)$ with $\left(\begin{array}{l}p_{j} \\ q_{j}\end{array}\right)=p_{j} ! / q_{j} !\left(p_{j}-q_{j}\right) !$.

We shall denote once for all by $\sigma$ vectors $\left(\sigma_{1}, \ldots \ldots, \sigma_{n}\right)$ whose components are 0 or 1 and adopt the following conventions: (v) $(-1)^{|\sigma|}=(-1)^{\sigma_{1}+\ldots+\sigma_{n}}$; (vi) $x^{\sigma}=\left((-1)^{\sigma_{1}} x_{1}, \ldots \ldots,(-1)^{\sigma_{n}} x_{n}\right)$ for any vector $x$; (vii) $k^{\sigma}=\left((-1)^{\sigma_{1}} k_{,} \ldots\right.$ $\ldots,(-1)^{\sigma_{n}} k$ ) for any integer $k$; (viii) $R_{\sigma}^{n}=\left\{x \in R^{n}: x^{\sigma} \geqq 0\right\}$; (ix) $C_{\sigma, \alpha}^{n}$ $=\left\{z \in C^{n}:(-1)^{\sigma_{1} \mathscr{F} z_{1}}>\alpha, \ldots \ldots,(-1)^{\sigma_{n}} \mathcal{F} z_{n}>\alpha\right\}$ with $\alpha>0$ and $(\mathrm{x}) \Delta_{\sigma, \alpha}$ is the path of integration $\left(-\infty+i(-1)^{\sigma_{1}} \alpha, \infty+i(-1)^{\sigma_{1}} \alpha\right) \times \ldots \ldots \times(-\infty+$ $\left.i(-1)^{\sigma_{n}} \alpha, \infty+i(-1)^{\sigma_{n}} \alpha\right)$, oriented from $-\infty$ to $+\infty$. Finally $V_{x}$ denotes the horizontal band in $C^{n}$ defined by $V_{\alpha}=\left\{z \in C^{n}:\left|\mathscr{F} z_{1}\right| \leqq \alpha, \ldots, .,\left|\mathscr{F} z_{n}\right| \leqq \alpha\right\}$ with $\alpha>0$.

1. The basic spaces $H$ and $\Lambda_{\infty}$. Let $H$ be the space of all $C^{\infty}$-functions $\varphi(x)$ in $R^{n}$ such that $\exp (k|x|) D^{p} \varphi(x)$ is bounded in $R^{n}$ for any $k$ and $p$. We define in $H$ semi-norms 


$$
\|\boldsymbol{\varphi}\|_{k}=\sup _{0 \leqq|p| \leqq k, x} \exp (k|x|)\left|D^{p} \boldsymbol{\varphi}(x)\right|, k=0,1,2, \ldots \ldots .
$$

Then $H$ is a Hausdorff locally convex metrizable space.

Let $\Gamma$ be a set of continuous functions on $R^{n}$ such that, for 'any compact subset $K \subset R^{n}$, there exists a member $\gamma \in \Gamma$ which never vanishes on $K$. We say that a function $\varphi \in(\mathscr{E})$ satisfies the condition of growth defined by $\Gamma$ if, for any $p$ and for any $\gamma \in \Gamma$, the function $\gamma(x) D^{p} \phi(x)$ is bounded in $R^{n}$. Thus the space $H$ consists of all functions in (E) satisfying the condition of growth defined by the class $\Gamma_{0}=\{\exp (k|x|): k=0,1,2, \ldots \ldots\}$ or equivalently by the class $\Gamma_{0}^{\prime}=\left\{\exp \left(k^{\sigma} \cdot x\right)\right\}$ where $k=0,1,2, \ldots \ldots$ and $\sigma$ varies over all vectors whose components are 0 or 1 .

PROPOSITION 1. The space $H$ is a Fréchet nuclear space and therefore completely reflexive.

PROOF. The mappings $\Phi_{k \sigma}(k=0,1,2, \ldots \ldots$ and any $\sigma)$, defined by $\Phi_{k \sigma}(\boldsymbol{\phi})$ $=\exp \left(k^{\sigma} \cdot x\right) \boldsymbol{\varphi}(x)$, are continuous linear mappings of $H$ into (め). In fact, if $\boldsymbol{\phi}_{\nu}$ $\rightarrow 0$ in $H$, then $\exp ((k+1)|x|) D^{p} \boldsymbol{\phi}_{\nu}(x) \rightarrow 0$ uniformly in $R^{n}$. Thus, for any polynomial $P(x), P(x) \exp \left(k^{\sigma} \cdot x\right) D^{p} \boldsymbol{\varphi}_{\nu}(x) \rightarrow 0$ uniformly in $R^{n}$ and therefore $P(x)$ $\rightarrow \cdot D^{p}\left[\exp \left(k^{\sigma} \cdot x\right) \boldsymbol{\varphi}_{\nu}(x)\right] \rightarrow 0$ uniformly in $R^{n}$. As $p$ is arbitrary, $\Phi_{k \sigma}\left(\boldsymbol{\varphi}_{\nu}\right) \rightarrow 0$ in $(\varnothing)$.

Now, suppose that $\Phi_{k \sigma}\left(\varphi_{\nu}\right) \rightarrow 0$ in (\&) for any $k=0,1,2, \ldots \ldots$ and any $\sigma$. Then it is easy to show that $\exp \left(k^{\sigma} \cdot x\right) D^{p} \boldsymbol{\varphi}_{\nu}(x) \rightarrow 0$ uniformly in $R^{n}$. Thus $\boldsymbol{\varphi}_{\nu}$ $\rightarrow 0$ in $H$.

Moreover, if $\boldsymbol{\varphi} \in(\mathscr{E})$ and $\Phi_{k \sigma}(\boldsymbol{\varphi}) \in(\wp)$ for any $k=0,1,2, \ldots \ldots$ and any $\sigma$, then $\boldsymbol{\varphi} \in H$. Hence $H$ is the projective limit of $(\delta)$ with respect to the mappings $\Phi_{k \sigma}$. Since (\&) is nuclear, $H$ is also nuclear by $[1$; Chap. II, Cor. 1 to Th. 9] or [4; Exposé 18, Cor. 1 to Prop. 7].

Let $\boldsymbol{\varphi}_{\nu}$ be any Cauchy sequence in $H$. Then the sequence $\boldsymbol{\varphi}_{\nu}=\Phi_{0}\left(\boldsymbol{\varphi}_{\nu}\right)$ converges to some $\varphi(x) \in(\&)$ under the topology of $(\delta)$. It is then easy to see that $\boldsymbol{\varphi} \in H$ and $\boldsymbol{\varphi}_{\nu} \rightarrow \boldsymbol{\phi}$ in $H$. Hence $H$ is complete.

In any Fréchet nuclear space, a bounded set $B$ is contained in the closed circular convex envelope of a convergent sequence and therefore relatively compact. Thus the space $H$ is completely reflexive. q. e. d.

PROPOSITION 2. The space $H$ is of type $\mid \psi^{\infty}$ in the sense of Schwartz [4], i. e., it satisfies the following conditions :

$\left(H_{1}\right) H$ is the space of $\phi \in(\mathscr{E})$ satisfying the condition of growth defined by $\Gamma_{0}$.

$\left(\mathrm{H}_{2}\right) \mathrm{H}$ is a Hausdorff complete locally convex space and the injections $(\mathscr{D}) \rightarrow H \rightarrow(\mathscr{E})$ are continuous.

$\left(H_{3}\right) A$ subset $B \subset H$ is bounded if and only if, for any $\gamma \in \Gamma_{0}$ and $p$, the set of numbers $\gamma(x) D^{p} \phi(x), \phi \in B, x \in R^{n}$, is bounded. 
$\left(H_{4}\right)$ On any bounded set $B \subset H$, the topology induced by $H$ coincides with the one induced by (E).

PROOF. $\left(H_{1}\right)$ and $\left(H_{3}\right)$ are obvious from the definition of $H$. The injections $(\mathscr{D}) \rightarrow H \rightarrow(\mathscr{E})$ are clearly continuous. Thus, in view of Prop. $1,\left(H_{2}\right)$ is satisfied. If $B$ is bounded and closed in $H$, then it is compact and therefore compact with respect to the topology induced by $(\mathscr{E})$. Hence the two topologies coincide and $\left(H_{4}\right)$ is fulfilled. q. e. d.

PROPOSITION 3. The dual of $H$ is the space $\Lambda_{\infty}$ of all distributions $T$ of exponential type such that

$$
T=\left(\partial^{k} / \partial x^{k}\right)[\exp (k|x|) f(x)],
$$

where $k$ is an integer $\geqq 0$ and $f(x)$ is a bounded continuous function. $\Lambda_{\infty}$ is a nuclear space of type $b_{c}^{\prime \prime}$ in the sense of Schwartz [4] under the strong topology.

PROOF. It is clear that a distribution $T$ of the form (2) defines a continuous linear functional on $H$. Conversely, let $T$ be any distribution defining a continuous linear functional on $H$. Then the set of distributions $\left\{\exp \left(-k^{\prime}|u|\right)\right.$ $\left.{ }^{-} \tau_{u}\left(T_{x}\right): u \in R^{n}\right\}$ is bounded in $\left(\mathscr{D}^{\prime}\right)$ for some $k^{\prime}>0$. To see this, we notice firstly that the set of semi-norms (1) is equivalent to the system of semi-norms

$$
\|\boldsymbol{\phi}\|_{k}^{\prime}=\sup _{0 \leqq|p| \leqq k, x, \sigma}\left|D^{p}\left[\exp \left(k^{\sigma} \cdot x\right) \boldsymbol{\phi}(x)\right]\right|, k=0,1,2, \ldots \ldots .
$$

Since $T$ is continuous in $H$, there exists an integer $k^{\prime}>0$ and an $\varepsilon>0$ such that $\|\boldsymbol{\phi}\|_{l}^{\prime} \leqq \varepsilon$ for $l=0,1, \ldots \ldots, k^{\prime}$, imply $|T(\phi)| \leqq 1$. For any $\phi \in(\mathscr{D})$, we have $\left[\exp \left(-k^{\prime}|u|\right) \tau_{u}\left(T_{x}\right)\right] \boldsymbol{\varphi}(x)=\exp \left(-k^{\prime}|u|\right) T_{x}(\boldsymbol{\phi}(x+u))$. On the other hand,

$$
\begin{aligned}
\|\boldsymbol{\phi}(x+u)\|_{l}^{\prime} & =\sup _{|\leqq| p \mid \leqq l, x, \sigma}\left|D^{p}\left[\exp \left(l^{\sigma} \cdot x\right) \boldsymbol{\phi}(x+u)\right]\right| \\
& =\sup \exp \left(-l^{\sigma} \cdot u\right)\left|D^{p}\left[\exp \left(l^{\sigma} \cdot(x+u)\right) \boldsymbol{\phi}(x+u)\right]\right| \\
& \leqq \exp (l|u|) \sup \left|D^{p}\left[\exp \left(l^{\sigma} \cdot x\right) \boldsymbol{\phi}(x)\right]\right| \\
& =\exp (l|u|)\|\boldsymbol{\phi}\|_{l}^{\prime} \leqq \exp \left(k^{\prime}|u|\right)\|\boldsymbol{\phi}\|_{l}^{\prime} .
\end{aligned}
$$

Thus we have

$$
\begin{aligned}
& \left|\left[\exp \left(-k^{\prime}|u|\right) \tau_{u}\left(T_{x}\right)\right] \phi(x)\right|=\exp \left(-k^{\prime}|u|\right)\left|T_{x} \varphi(x+u)\right| \\
& \leqq \exp \left(-k^{\prime}|u|\right) \max _{0 \leqq I \leqq k^{\prime}}\left\{\varepsilon^{-1} \exp \left(k^{\prime}|u|\right)\|\varphi\|_{i}^{\prime}\right\} \\
& \leqq \varepsilon^{-1} \max _{0 \leqq I \leqq k^{\prime}}\left\{\|\boldsymbol{\phi}\|_{l}^{\prime}\right\} \text {. }
\end{aligned}
$$

As $\phi$ is arbitrary, this shows that $\left\{\exp \left(-k^{\prime}|u|\right) \tau_{u}\left(T_{x}\right): u \in R^{n}\right\}$ is bounded in $\left(\mathscr{D}^{\prime}\right)$. By a theorem of Schwartz [2; Chap. VI, Th. XXII], there exist an integer $m \geqq 0$ and a sufficiently small compact neighborhood $K$ of the origin of 
$R^{n}$ such that, for any $\boldsymbol{\phi} \in\left(\mathscr{D}_{k}^{m}\right),\left\{\exp \left(-k^{\prime}|u|\right) \tau_{u}(T * \phi): u \in R^{n}\right\}$ forms a family of bounded continuous functions on some relatively compact open set $\boldsymbol{\Omega}$. Therefore, $\exp \left(-k^{\prime}|x|\right)(T * \boldsymbol{\varphi})(x)$ is continuous and bounded in $R^{n}$. We know that the elementary solution $E$ for the iterated Laplace equation $\Delta^{N} E=\delta$ is $m$-times continuously differentiable for large $N$. Then, for any $\gamma(x) \in\left(\mathscr{D}_{K}\right), \gamma E$ belongs to $\left(\mathscr{D}_{K}^{m}\right)$ and $\delta=\Delta^{N}(\gamma E)-\zeta$ where $\zeta \in(\mathscr{D})$. Hence $T=\Delta^{N}(\gamma E * T)-\zeta * T$ and therefore $T$ is a (finite) sum of distributions of the form $D^{p}\left[\exp \left(k^{\prime \prime}|x|\right) f(x)\right]$, $f(x)$ being bounded and continuous. Now it is easy to show that $T$ can be reduced to the form (2).

Since $H$ is a Fréchet nuclear space, bounded sets and relatively compact sets are the same in $H$ and thus the strong topology $\tau_{b}$ and the topology $\tau_{c}$ of compactconvergence coincide in $H^{\prime}=\Lambda_{\infty}$. Thus $\Lambda_{\infty}$ is of type $\not \phi_{c}^{\prime \infty}$. As the dual of a Fréchet nuclear space $H$, the space $\Lambda_{\infty}$ is also nuclear. q. e. d.

Let $B$ be a bounded set $\subset \Lambda_{\infty}$. Then $B$ is equicontinuous and therefore there exists an integer $k^{\prime} \geqq 0$ such that $\|\boldsymbol{\varphi}\|_{l}^{\prime} \leqq \varepsilon\left(l=0,1, \ldots \ldots, k^{\prime}\right)$ imply $|T(\boldsymbol{\varphi})| \leqq 1$ for any $T \in B$. Then, by the argument used above, we have the following

COROLLARY. A set $B \subset \Lambda_{\infty}$ is bounded if and only if there exist an integer $k \geqq 0$ and a number $M>0$ such that $B$ is contained in the set of distributions $T=\left(\partial^{k} / \partial x^{k}\right)[\exp (k|x|) g(x)]$ with $g(x)$ bounded and continuous satisfying $\sup _{x_{\in} R^{n}}|g(x)| \leqq M$.

2. Fourier transform of $H$ and $\Lambda_{\infty}$. The spaces $\mathscr{S}$ and $U$. We shall construct the Fourier transform of $H$. Let $\phi \in H$ and put

$$
f(z) \equiv(\mathcal{F} \boldsymbol{\phi})(z)=(2 \pi)^{-n / 2} \int \cdots \cdots \int_{R^{n}} \exp (-i z \cdot x) \boldsymbol{\varphi}(x) d x,
$$

for any $z \in C^{n}$. Since $\boldsymbol{\varphi}$ satisfies the condition of growth defined by $\Gamma_{0}$, the integral converges uniformly in any horizontal bands $V_{k}, k=1,2, \ldots \ldots$, and therefore $f(z)$ is an entire function. We have

$$
\begin{aligned}
z^{m} f(z) & =(2 \pi)^{-n / 2} \int \cdots \cdots \int_{R^{n}} i^{m m}\left(\partial^{m} / \partial x^{m}\right)[\exp (-i z \cdot x)] \boldsymbol{\varphi}(x) d x \\
& =(2 \pi)^{-n / 2}(-i)^{m n} \int \cdots \cdots \int_{R^{n}} \exp (-i z \cdot x)\left(\partial^{m} / \partial x^{m}\right) \boldsymbol{\varphi}(x) d x
\end{aligned}
$$

which converges uniformly in any bands $V_{k}(k=1,2, \ldots \ldots)$ and

$$
\begin{aligned}
\left|z^{m} f(z)\right| & \leqq(2 \pi)^{-n / 2} \int \ldots \ldots \int_{R^{n}} \exp (+\mathscr{F} z \cdot x)\left|\left(\partial^{m} / \partial x^{m}\right) \varphi(x)\right| d x \\
& \leqq(2 \pi)^{-n / 2} \int \cdots \cdots \int_{R^{n}} \exp (k|x|)\left|\left(\partial^{m} / \partial x^{m}\right) \varphi(x)\right| d x
\end{aligned}
$$




$$
\begin{aligned}
& \leqq(2 \pi)^{-n / 2} \int \cdots \cdots \int_{R^{n}} \exp (k|x|) \exp \left(-m^{\prime}|x|\right)\|\varphi\|_{m^{\prime} n} d x \\
& \leqq 2^{n}(2 \pi)^{-n / 2}\left(m^{\prime}-k\right)^{-n}\|\varphi\|_{m^{\prime} n}
\end{aligned}
$$

for $z \in V_{k}$, where $m^{\prime}>\max (m, k)$. As $m$ is arbitrary, $f(z)$ is rapidly decreasing in any horizontal bands.

Conversely, suppose that $f(z)$ is an entire function decreasing rapidly in any horizontal bands $V_{k}$ and put

$$
\varphi(x) \equiv(\widetilde{\mathcal{F}} f)(x)=(2 \pi)^{-n / 2} \int \cdots \cdots \int_{R^{n}} \exp (i x \cdot u) f(u) d u .
$$

Then, for any $p$, we have

$$
\begin{aligned}
D^{p} \phi(x) & =(2 \pi)^{-n / 2} D^{p} \int \ldots \int_{R^{n}} \exp (i x \cdot u) f(u) d u \\
& \left.=(2 \pi)^{-n / 2} \int \ldots \int_{R^{n}} \exp ^{\prime} i x \cdot u\right)(i u)^{p} f(u) d u \\
& =(2 \pi)^{-n / 2} \int_{-\infty+i v_{1}}^{\infty+i v_{1}} \ldots \int_{-\infty+i v_{n}}^{\infty+i v_{n}} \exp (i x \cdot \zeta)(i \zeta)^{p} f(\zeta) d \zeta \\
(4) \quad & =(2 \pi)^{-n / 2} D_{x}^{p} \int \cdots \int_{R^{n}} \exp (i x \cdot(u+i v)) f(u+i v) d u \\
& =(2 \pi)^{-n / 2} D_{x}^{p}\left[\exp (-v \cdot x) \int \cdots \int_{R^{n}} \exp (i x \cdot u) f(u+i v) d u\right] \\
& =(2 \pi)^{-n / 2} \sum_{0 \leqq q \leqq p}\left(\begin{array}{l}
p \\
q
\end{array}\right) D_{x}^{p-q} \exp (-v \cdot x) D_{x}^{q} \int \ldots \int_{R^{n}} \exp (i x \cdot u) f(u+i v) d u \\
& =\exp (-v \cdot x)\left[(2 \pi)^{-n / 2} \sum_{0 \leqq q \leqq p}\left(\begin{array}{l}
p \\
q
\end{array}\right)(-v)^{p-q} \int \cdots \int_{R^{n}} \exp (i x \cdot u)(i u)^{q} f(u+i v) d u\right] .
\end{aligned}
$$

Since the function in the bracket is continuous and bounded in $R^{n}$, we know, by setting $v=\left(k_{1}, \ldots \ldots, k_{n}\right), k_{j}= \pm 1, \pm 2, \ldots \ldots$, that $\boldsymbol{\varphi}$ satisfies the condition of growth defined by $\Gamma_{0}$, i. e., $\phi \in H$. Thus we have the first part of

PROPOSITION 4. The Fourier transform of the space $H$ is the space 5 of entire functions rapidly decreasing in any horizontal bands. The algebraic isomorphism becomes topogical if we define a topology in 5 by semi-norms

$$
p_{k}(f)=\sup _{z_{\epsilon} V_{k}}\left|z^{k} f(z)\right|, \quad k=0,1,2, \ldots \ldots .
$$

PROOF OF THE SECOND PART. Suppose that $\boldsymbol{\varphi}_{\nu} \rightarrow 0$ in $H$. Then $\left\|\boldsymbol{\varphi}_{\nu}\right\|_{m} \rightarrow 0$ for any $m \geqq 0$. Setting $m=k$ and $f(z)=f_{\nu}(z)=\left(\mathcal{F} \boldsymbol{\varphi}_{\nu}\right)(z)$ in (3), we have 


$$
p_{k}\left(f_{\nu}\right)=\sup _{z_{\mathrm{\epsilon}} V_{k}}\left|z^{k} f_{\nu}(z)\right| \leqq 2^{n}(2 \pi)^{-n / 2}\left(m^{\prime}-k\right)^{-n}\left\|\boldsymbol{\varphi}_{\nu}\right\|_{m^{\prime} n} \rightarrow 0
$$

where $m^{\prime}>k$. Thus, $p_{k}\left(f_{\nu}\right) \rightarrow 0$ for any $k=0,1,2, \ldots \ldots$ and therefore $f_{\nu} \rightarrow 0$ in $\mathcal{S}$. Now, for any $k=1,2, \ldots \ldots$ and any index $p$ with $p_{i} \leqq k$, we have

$$
\begin{aligned}
& \left|\int \cdots \int_{R^{n}} \exp (i x \cdot u)(i u)^{p} f\left(u+i k^{\sigma}\right) d u\right| \leqq \int \cdots \int_{R^{n}}\left|u^{p}\right|\left|f\left(u+i k^{\sigma}\right)\right| d u \\
& \quad \leqq \int \cdots \int_{R^{n}}\left|\left(u+i k^{\sigma}\right)^{p}\right|\left|f\left(u+i k^{\sigma}\right)\right| d u \\
& \quad=\int \cdots \int_{R^{n}}\left|\left(u+i k^{\sigma}\right)^{-2}\right|\left|\left(u+i k^{\sigma}\right)^{p_{+2}}\right|\left|f\left(u+i k^{\sigma}\right)\right| d u \\
& \quad \leqq \int \cdots \int_{R^{n}} \Pi_{j=1}^{n}\left(u_{j}^{2}+k^{2}\right)^{-1} d u \cdot p_{k+2}(f)=\pi^{n} k^{-n} p_{k+2}(f)
\end{aligned}
$$

where $p+2=\left(p_{1}+2, p_{2}+2, \ldots \ldots, p_{n}+2\right)$.

Thus it follows from (4) that

$$
\begin{aligned}
& \left|\exp \left(k^{\sigma} \cdot x\right) D^{p} \boldsymbol{\phi}(x)\right| \\
& \quad \leqq(2 \pi)^{-n / 2}\left|\sum_{0 \leqq q \leqq p}\left(\begin{array}{c}
p \\
q
\end{array}\right)\left(-k^{\sigma}\right)^{p_{-} q} \int \cdots \int_{R^{n}} \exp (i x \cdot u)(i u)^{q} f\left(u+i k^{\sigma}\right) d u\right| \\
& \quad \leqq(2 \pi)^{-n / 2} \pi^{n} k^{-n}\left[\sum_{0 \leqq q \leqq p}\left(\begin{array}{l}
p \\
q
\end{array}\right) k^{|p-q|}\right] p_{k+2}(f) .
\end{aligned}
$$

Therefore, for $k=1,2, \ldots \ldots$,

$$
\|\boldsymbol{\varphi}\|_{k}=\sup _{0 \leqq|p| \leqq k, x}\left|\exp (k|x|) D^{p} \boldsymbol{\varphi}(x)\right| \leqq c_{k} p_{k+2}(f),
$$

where $c_{k}$ is a constant depending only on $k$ and the dimension $n$. This proves that, if $f_{\nu} \rightarrow 0$ in $\mathfrak{S}_{2}$, then $\boldsymbol{\varphi}_{\nu} \rightarrow 0$ in $H$. Hence the proposition is proved.

Now we shall describe explicitly the dual $\mathfrak{S}^{\prime}$ of $\mathcal{F}_{\text {. }}$. Decompose $T \in \Lambda_{\infty}$ as follows :

$$
T=\sum_{\sigma}(-1)^{|\sigma|} T^{\sigma}
$$

where $T^{\sigma} \in \Lambda_{\infty}$ and the carrier of $T^{\sigma}$ is contained in $R_{\sigma}^{n}$. For example, if $T$ $=D^{p}\left[\exp \left(k^{\prime}|x|\right) g(x)\right], g(x)$ being bounded and continuous, then we may put $T^{\sigma}$ $=(-1)^{|\sigma|} D^{p}\left[\exp \left(k^{\prime}|x|\right) g(x) Y\left(x^{\sigma}\right)\right]$ where $Y(x)$ is the $n$-dimensional Heaviside's function. For any such decomposition, we set

$$
F^{\sigma}(z)=\left[\overline{\mathcal{F}} T^{\sigma}\right](z)=(2 \pi)^{-n / 2}<\exp (i z \cdot x), T_{x}^{\sigma}>.
$$

Then there exists a number $k>0$ such that each $F^{\sigma}(z)$ is analytic in $C_{\sigma, k}^{n}$ and $F^{\sigma}(z) / z^{k}$ is bounded and continuous in $\overline{C_{\sigma, k}^{n}}$. 
Conversely, for such a function $F(z)=\sum_{\sigma} F^{\sigma}(z)$, define distributions $T^{\sigma}$ by

$$
T_{y}^{\sigma}=\left(\partial^{k+2} / \partial y^{k+2}\right)\left[(2 \pi)^{-n / 2} \int \cdots \int_{\Delta_{\sigma, k}} \exp (-i y \cdot \zeta) F^{\sigma}(\zeta)(-i \zeta)^{-(k+2)} d \zeta \cdot Y\left(y^{\sigma}\right)\right]
$$

and a distribution $T$ by the formula (6). Then $T \in \Lambda_{\infty}$ and the functions $G^{\sigma}(z)$ which are associated, by (7), with this decomposition are nothing but the given functions $F^{\sigma}(z)$. In fact, for $z \in C_{\sigma, k}^{n}$,

$$
\begin{aligned}
G^{\sigma}(z) & \equiv<(2 \pi)^{-n / 2} \exp (i z \cdot y), T_{y}^{\sigma}> \\
& =(-i z)^{k+2}\left[(2 \pi)^{-n} \int \cdots \int_{\Delta_{\sigma, k}} F^{\sigma}(\zeta)(-i \zeta)^{-(k+2)} d \zeta\left[\int \cdots \int_{R^{n}} \exp (-i y \cdot(z-\zeta)) d y\right]\right] \\
& =(-i z)^{k+2}\left[(2 \pi i)^{-n} \int \cdots \int_{\Delta_{\sigma, k}} F^{\sigma}(\zeta)(-i \zeta)^{-(k+2)}(-1)^{|\sigma|}(\zeta-z)^{-1} d \zeta\right] \\
& =(-i z)^{k+2}\left[F^{\sigma}(z)(-i z)^{-(k+2)}\right]=F^{\sigma}(z) .
\end{aligned}
$$

If we denote by $a_{\omega}$ the space of all functions $F(z)$ such that (i) $F(z)$ is analytic in $\left\{z \in C^{n}:\left|\mathscr{F} z_{1}\right|>k, \ldots \ldots,\left|\mathscr{F} z_{n}\right|>k\right\}$ and (ii) $F(z) / z^{k}$ is bounded continuous in $\left\{z \in C^{n}:\left|\mathscr{F} z_{1}\right| \geqq k, \ldots \ldots,\left|\mathscr{F} z_{n}\right| \geqq k\right\}, k$ depending on $F(z)$, then we have shown that the mapping $f: F(z) \rightarrow T$, defined by the formulae (8) and (6), is a mapping of $a_{\omega}$ onto $\Lambda_{\infty}$.

Let $F \in a_{\omega}$ and $T=\mathcal{F} F$. Then there exists a decomposition (6) such that $F(z)=\sum_{\sigma} F^{\sigma}(z)$ with $F^{\sigma}(z)=\left[\overline{\mathcal{F}} T^{\sigma}\right](z)$ for $z \in C_{\sigma, k}^{n}$ where $k$ is any integer $>0$ having the properties (i) and (ii) above. We may write $T_{y}^{\sigma}=\left(\partial^{k} / \partial y^{k}\right)$. $\left[\exp (k|y|) h^{\sigma}(y)\right]$ where $h^{\sigma}(y)$ is bounded, continuous in $R_{\sigma}^{n}$ and vanishes outside of $R_{\sigma}^{n}$. Then, for any $f \in \mathscr{F}_{\text {, }}$

$$
\begin{aligned}
\left\langle\overline{\mathcal{F}} f, T^{\sigma}\right\rangle & =\left\langle(2 \pi)^{-n / 2} \int \cdots \int_{R^{n}} \exp (i y \cdot x) f(x) d x, T_{y}^{\sigma}\right\rangle \\
& =(2 \pi)^{-n / 2}\left\langle\int \cdots \int_{\Delta_{\sigma, k+1}} \exp (i y \cdot \zeta) f(\zeta) d \zeta,\left(\partial^{k} / \partial y^{k}\right)\left[\exp (k|y|) h^{\sigma}(y)\right]\right\rangle \\
& =(2 \pi)^{-n / 2} \int \cdots \int_{R_{\sigma}^{n}}\left[\int \cdots \int_{\Delta_{\sigma, k+1}}(-i \zeta)^{k} \exp (i y \cdot \zeta) f(\zeta) d \zeta\right] \exp (k|y|) h^{\sigma}(y) d y \\
& =\int \cdots \int_{\Delta_{\sigma, k+1}} f(\zeta)\left[(2 \pi)^{-n / 2}(-i \zeta)^{k} \int \cdots \int_{R_{\sigma}^{n}} \exp (k|y|+i \zeta \cdot y) h^{\sigma}(y) d y\right] d \zeta \\
& =\int \cdots \int_{\Delta_{\sigma, k+1}} f(\zeta) F^{\sigma}(\zeta) d \zeta \\
& =\int \cdots \int_{\Delta_{\sigma, k}} f(\zeta) F(\zeta) d \zeta,
\end{aligned}
$$


from which follows

$$
\begin{aligned}
& \langle f, F\rangle=\langle f, \overline{\mathcal{F}} T\rangle=\langle\overline{\mathcal{F}} f, T\rangle=\sum_{\sigma}(-1)^{|\sigma|}\left\langle\overline{\mathcal{F}} f, T^{\sigma}\right\rangle \\
& =\sum_{\sigma}(-1)^{|\sigma|} \int \cdots \int_{\Delta_{\sigma, k}} f(\zeta) F(\zeta) d \zeta \\
& =\int \cdots \int_{L_{k}} f(\zeta) F(\zeta) d \zeta
\end{aligned}
$$

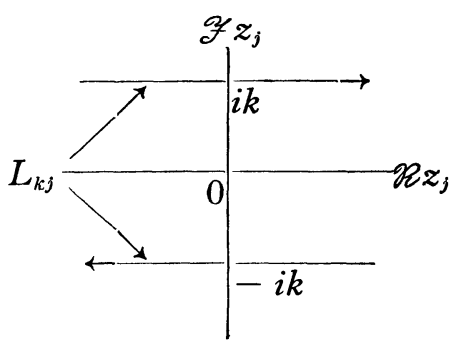

Fig. 1

where $L_{k}$ is the product of pathes $L_{k j}(j=1,2, \ldots \ldots, n)$ defined by Fig. 1 .

It is now clear that $\langle f, F\rangle=0$ for all $f \in \mathfrak{S}$ if and only if $F$ belongs to the kernel of the mapping $\mathcal{F}: a_{\omega} \rightarrow \Lambda_{\infty}$. Let $\Pi$ be the kernel of $\mathcal{F}$. Obviously, any element in $a_{\omega}$, which is a polynomial in one of the variables $z_{1}, \ldots \ldots, z_{n}$ that is,

$$
\sum_{s} z_{j}^{s} G_{s}\left(z_{1}, \ldots \ldots, z_{j-1}, z_{j+1}, \ldots \ldots, z_{n}\right)
$$

where $G_{s}$ are functions in $a_{\omega}$ with respect to $\left(z_{1}, \ldots \ldots, z_{j-1}, z_{j+1}, \ldots \ldots, z_{n}\right)$, belongs to $\Pi$. Conversely, we can prove readily that $\Pi$ is the subspace of $a_{\omega}$ generated by all such polynomials. Thus, summing up these considerations, we obtain

PROPOSITION 5. The dual Sू' of $\mathfrak{S}^{2}$ is algberaically isomorphic with the space $U$ which is the quotient space of $a_{\omega}$ by $\Pi$. If $F(z)$ is any representative of an element $u \in \mathcal{U}$ and $k$ is determined by (i) and (ii), then the duality between $U$ and $\mathfrak{S}$ is given by

$$
<f, u>=\int \cdots \int_{\Sigma_{k}} f(\zeta) F(\zeta) d \zeta \quad \text { for } f \in S_{2}
$$

The distribution $T=\mathcal{F} u \in \Lambda_{\infty}$ corresponding to $u$ is then expressed by (8) and (6).

Any element in $\mathcal{U}$ is called a tempered ultra-distribution in the $n$-dimensional space. In view of Cor. to Prop. 3, we have 
PROPOSITION 6. A set $B \subset \mathcal{U}$ is bounded if and only if there exist an integer $k \geqq 0$ and a number $M>0$ such that each element $u \in B$ has a representative $F(z)$ satisfying (i) $F(z)$ is analytic in $\left\{z \in C^{n}:\left|\mathscr{F} z_{1}\right|>k, \ldots \ldots\right.$, $\left.\left|\mathscr{F} z_{n}\right|>k\right\}$, continuous in $\left\{z \in C^{n}:\left|\mathscr{F} z_{1}\right| \geqq k, \ldots \ldots,\left|\mathscr{F} z_{n}\right| \geqq k\right\}$ and (ii) $\left|F(z) / z^{k}\right| \leqq M$ in the latter set.

Since the strong dual $\mathcal{U}$ of $\mathfrak{S}_{\mathrm{C}}$ is bornological, a convex circular subset of I is a neighborhood of the origin if and only if it absorbs all bounded subsets of $U$. Thus we get an explicit description of the strong topology for U. Denoting by $B_{k}$ the subset of $\mathcal{U}$, each element of which has a representative $F(z)$ satisfying (i) and (ii) of Prop. 6 with $M=1$, a basis of neighborhoods of zero in $\mathscr{Y}$, then consists of $\Gamma_{k=1}^{\infty}\left(\varepsilon_{k} B_{k}\right)$ where $\varepsilon_{k}$ is any sequence of positive numbers $\Gamma_{k=1}^{\infty}$ denotes the convex circular envelope of $\varepsilon_{k} B_{k}$ 's. In other words, we may say

PROPOSITION 7. The strong dual as is the inductive limit of Banach spaces $\mathfrak{U}_{B_{k}}$, which are the subspace of $U_{l}$ generated by $B_{k}$ with the unit ball $B_{k}$.

REMARK. It is clear that the topological and algebraical structure of $\mathscr{U}$, stated in Prop. 7, is the same as that of Sebastião e Silva [3] when $n=1$.

An extension of ultra-distributions of exponential type to $n$-dimensional space is possible, which was also observed by Prof. Sebastião e Silva in a letter to the author.

3. An application. The multiplication in $\because$. Since $\Lambda_{\infty}$ contains the space $\left(\delta^{\prime}\right)$ of Schwartz's tempered distributions and $\mathcal{F}$ is an automorphism of $\left(\delta^{\prime}\right)$, we may regard $\left(\delta^{\prime}\right)$ as a subspace of $U$, the characterization of which was obtained by Sebastião e Silva [3; Prop. 12.1]. We can obtain a similar characterization for arbitrary $n$. It is well known that $\alpha \in\left(\bar{\sigma}_{n}\right)$ defines a continuous mapping $[\alpha]: S \rightarrow \alpha S$ of $\left(\varnothing^{\prime}\right)$ into itself. For the mutiplication-operation in $\mathscr{U}$, we have the following

PROPOSITION 8. The mapping $[\alpha]\left(\alpha \in\left(\mathscr{\odot}_{M}\right)\right)$, defined in $\left(8^{\prime}\right) \subset U$, is continuously extendable to a continuous linear mapping of U into itself, if and only if $\alpha(x)$ is extendable over $C^{n}$ as an entire function slowly increasing in any horizontal bands.

PROOF. The "if"-part was obtained by Sebastião e Silva [3; Prop. 15. 1]. Indeed, if $\alpha \in\left(\mathscr{O}_{r}\right)$ can be extended to an entire function $\bar{\alpha}$ satisfying the condition, then it is easy to see that $f \rightarrow \tilde{\alpha} f(f \in \mathcal{S})$ defines a continuous operation in $\mathfrak{S}_{\text {s }}$ so that $\tilde{\alpha}$ provides a continuous multiplication in $\mathfrak{H}^{\prime}=\mathscr{U}$.

In order to verify the "only if"-part, we notice firstly that $[\alpha]$ is continuously extendable to $\mathcal{U}$ if and only if the convolution operation $T \rightarrow A * T(A=\mathcal{F} \alpha \in$ 
$\left(\mathscr{O}^{\prime}\right)$ ), defined in $\left(\delta^{\prime}\right) \subset \Lambda_{\infty}$, is continuously extendable to a continuous linear mapping of $\Lambda_{\infty}$ into itself. Since $\Lambda_{\infty}$ is of type $\phi_{c}^{\prime \infty}$ by Prop. 3, we may apply a theorem. of Schwartz which we state as

LEMMA 1 ([4; Exposé 11, Theorem 1]). Let $E$ be a space of distributions, i. e., $E \subset\left(\mathscr{D}^{\prime}\right)$ and the injection of $E$ into ( $\left.\mathscr{D}^{\prime}\right)$ is continuous. Let if be a space of type $\phi^{\infty}$ and $\mathscr{O}^{\prime}\left(\mid \psi_{c}^{\prime}:\right.$ E) the space of continuous linear mappings of $\psi_{c}^{\prime}$ into $E$ which are convolution operations on $\left(\mathscr{E}^{\prime}\right) \subset \phi_{c}^{\prime}$. Then a distribution $A$ belongs to $\mathscr{O}^{\prime}\left(\phi_{c}^{\prime}: E\right)$ if and only if the function $\vec{A}: y \rightarrow \tau_{y}\left(A_{x}\right), y \in R^{n}$, belongs to $\widetilde{\psi}(E)$, the space of indefinitely differentiable functions $\vec{\phi}$ with values in $E$ such that $\left\langle\overrightarrow{\boldsymbol{\phi}}, e^{\prime}\right\rangle \in \mid \phi$ for any $\boldsymbol{e}^{\prime} \in E^{\prime}$.

Now, suppose that $[\alpha]$ is continuously extendable to $U$ into itself and therefore that the convolution operation defined by $A=\mathcal{F} \alpha$ is continuously extendable to $\Lambda_{\infty}$, i. e., $A \in \mathscr{O}_{c}^{\prime}\left(\Lambda_{\infty}: \Lambda_{\infty}\right)$. Thus, by Lemma 1 , the function $\vec{A}: y \rightarrow \tau_{y} A_{t}$ must belong to $\tilde{\phi}\left(\Lambda_{\infty}\right)$ where $\phi=H$. This means that, for any $k \geqq 0$ and $p$, the set $\left\{\exp (k|y|) D_{y}^{p} \vec{A}(y): y \in R^{n}\right\}$ is bounded in $\Lambda_{\infty}$ by [4; Exposé 10, Prop. 4]. Especially for $p=0$, the set of distributions $\left\{\exp (k|y|) \tau_{y}\left(A_{t}\right): y \in R^{n}\right\}$ is bounded in $\Lambda_{\infty}$ and à fortiori bounded in $\left(\mathscr{D}^{\prime}\right)$. Then, by an argument similar to that used in the proof of Prop. 3, we see that there exist continuous bounded functions $g_{1}(t), g_{2}(t)$ and an integer $N>0$, all depending on $A$ and $k$, such that

$$
A_{t}=\Delta^{N}\left[\exp (-k|t|) g_{1}(t)\right]+\exp (-k|t|) g_{2}(t) \text {. }
$$

It follows that $<(2 \pi)^{-n / 2} \exp (i z \cdot t), A_{t}>$ has a meaning for any $z \in V_{k}$ and

$$
\begin{aligned}
& \tilde{\alpha}(z) \equiv<(2 \pi)^{-n / 2} \exp (i z \cdot t), A_{t}> \\
&=(2 \pi)^{-n / 2}\left\{\left[-\left(z_{1}^{2}+\ldots \ldots+z_{n}^{2}\right)\right]^{N} \int \cdots \int_{R^{n}} \exp (i z \cdot t-k|t|) g_{1}(t) d t\right. \\
&\left.\quad+\int \ldots \int_{R^{n}} \exp (i z \cdot t-k|t|) g_{2}(t) d t\right\} .
\end{aligned}
$$

Since the integrals in the last expression represent analytic functions bounded in $V_{k-\varepsilon}(\varepsilon>0)$, the function $\tilde{\alpha}(z)$ is analytic and slowly increasing in the bands $V_{k-\epsilon}(\varepsilon>0)$. As $k$ is arbitrary, we have shown that $\tilde{\alpha}(z)$ is an entire function slowly increasing in any horizontal bands. Clearly $\tilde{\alpha}(x)=\alpha(x)$ for $x \in R^{n}$ and this completes the proof.

In the course of the proof of Prop. 8, we have obtained

PROPOSITION 9. A distribution $T$ belongs to $\operatorname{O}^{\prime}\left(\Lambda_{\infty}: \Lambda_{\infty}\right)$ if and only if, for any integer $k>0$, there exists a finite number of bounded continuous functions $g_{j}(x)$ such that $T$ is a (finite) sum of distribution-derivatives of $\exp (-k|x|) g_{i}(x)$. 
ADDED IN PROOF. Recently, the following paper has appeared : K. Yoshinaga, "On spaces of distributions of exponential growth," Bulletin of the Kyushu Institute of Technology (Math. \& Nat. Sci.), No.6, 1960. This paper treats, independently of ours, a problem related to the one discussed here, especially to the section 3 .

\section{REFERENCES}

[1] A. Grothendieck, Produits tensoriels topologiques et espaces nucléaires, Mem. Amer. Math. Soc., No.16, New York, 1955.

[2] L. SchWARTZ, Théorie des distributions, II, Paris, 1951.

[3] J. SEBASTIÃo E SILVA, Les fonctions analytiques comme ultra-distributions dans le calcul opérationnel, Math. Ann., 136(1958), 58-96.

[4] Séminaire Schwartz, 1953-54, Paris, 1954.

DEPARTMENT OF MATHEMATICS,

IBARAKI UNIVERSITY. 